\title{
LOCALLY POSITIVE NONLINEAR SYSTEMS
}

\author{
TADEUSZ KACZOREK* \\ * Institute of Control and Industrial Electronics \\ Warsaw University of Technology \\ ul. Koszykowa 75, 00-662 Warszawa, Poland \\ e-mail: kaczorek@isep.pw.edu.pl
}

\begin{abstract}
The notion of locally positive nonlinear time-varying linear systems is introduced. Necessary and sufficient conditions for the local positiveness of nonlinear time-varying systems are established. The concept of local reachability in the direction of a cone is introduced, and sufficient conditions for local reachability in the direction of a cone of this class of nonlinear systems are presented.
\end{abstract}

Keywords: local positiveness, time-varying, nonlinear system, local reachability in the direction of a cone

\section{Introduction}

Roughly speaking, positive systems are systems whose trajectories are entirely in the non-negative orthant $\mathbb{R}_{+}^{n}$ whenever the initial state and input are non-negative. Positive systems arise in the modelling of systems in engineering, economics, social sciences, biology, medicine and other areas (Alessandro and Santis, 1994; Berrman et al., 1989; Berrman and Plemmons, 1994; Farina and Rinaldi, 2000; Kaczorek, 2002; Rumchev and James, 1990; Rumchev and James, 1999). The single-input single-output externally positive and internally positive linear timeinvariant systems were investigated in (Berrman et al., 1989; Berrman and Plemmons, 1994; Farina and Rinaldi, 2000). The notions of externally positive and internally positive systems were extended to singular continuoustime and discrete-time and two-dimensional linear systems in (Kaczorek, 2002). The reachability and controllability of standard and singular internally positive linear systems were analysed in (Fanti et al., 1990; Klamka, 1998; Ohta et al., 1984; Valcher, 1996). The notions of weakly positive discrete-time and continuous-time linear systems were introduced in (Kaczorek, 2002; 2001). Recently, the positive two-dimensional (2D) linear systems were extensively investigated by Fornasini and Valcher (Valcher, 1996; 1997) and Kaczorek (2002).

Necessary and sufficient conditions for the external and internal positivities and sufficient conditions for the reachability of time-varying linear systems were established in (Kaczorek, 2001; Klamka and Kalinowski, 1998). The notion of the controllability of a dynamic system in the direction of a cone was introduced by Walczak (1990) and a sufficient condition for the local controllabil- ity of nonlinear systems was established.

In this paper the notion of local positiveness in the neighborhood of zero of nonlinear time-varying systems will be introduced and the necessary and sufficient conditions for the local positiveness will be established. The reachability of nonlinear time-varying systems will also be investigated. To the best of the author's knowledge, this class of locally positive nonlinear systems has not been considered yet.

\section{Preliminaries}

Let $\mathbb{R}_{+}^{n \times m}$ be the set of real matrices with non-negative entries and $\mathbb{R}_{+}^{n}:=\mathbb{R}_{+}^{n \times 1}$. Consider a nonlinear system described by the equations

$$
\begin{aligned}
& \dot{x}=f(x, u, t), \quad x\left(t_{0}\right)=x_{0}, \\
& y=h(x, u, t),
\end{aligned}
$$

where $\dot{x}=\mathrm{d} x / \mathrm{d} t, x \in \mathbb{R}^{n}, u \in \mathbb{R}^{m}$ and $y \in \mathbb{R}^{p}$ are the state, input and output vectors, respectively, and

$$
\begin{gathered}
f(x, u, t)=\left[\begin{array}{c}
f_{1}(x, u, t) \\
f_{2}(x, u, t) \\
\vdots \\
f_{n}(x, u, t)
\end{array}\right], \\
h(x, u, t)=\left[\begin{array}{c}
h_{1}(x, u, t) \\
h_{2}(x, u, t) \\
\vdots \\
h_{p}(x, u, t)
\end{array}\right]
\end{gathered}
$$


are $\mathbb{R}^{n}$ and $\mathbb{R}^{p}$-valued mappings defined on open sets. It is assumed that the functions $f_{1}(x, u, t), \ldots$, $f_{n}(x, u, t)$ and $h_{1}(x, u, t), \ldots, h_{p}(x, u, t)$ are smooth in their arguments, i.e., they are real-valued functions of $x_{1}, \ldots, x_{n}, u_{1}, \ldots, u_{m}, t$ with continuous partial derivatives of any order, where $x=\left[x_{1}, x_{2}, \ldots, x_{n}\right]^{T}, u=$ $\left[u_{1}, u_{2}, \ldots, u_{m}\right]^{T}$ and $T$ denotes transposition. It is also assumed that the system (1a) possesses a solution for any admissible input $u$.

Let

$$
f(0,0, t)=0, \quad h(0,0, t)=0, \forall t
$$

and

$$
\begin{aligned}
& \dot{x}=A(t) x+B(t) u+N_{f}(x, u, t), \\
& y=C(t) x+D(t) u+N_{h}(x, u, t),
\end{aligned}
$$

where

$$
\begin{aligned}
& A(t)=\left.\frac{\partial f}{\partial x}\right|_{\substack{x=0 \\
u=0}}=\left.\left[\begin{array}{ccc}
\frac{\partial f_{1}}{\partial x_{1}} & \ldots & \frac{\partial f_{1}}{\partial x_{n}} \\
\ldots \ldots & \ldots & \ldots \ldots \\
\frac{\partial f_{n}}{\partial x_{1}} & \cdots & \frac{\partial f_{n}}{\partial x_{n}}
\end{array}\right]\right|_{\substack{x=0 \\
u=0}}, \\
& B(t)=\left.\frac{\partial f}{\partial x}\right|_{\substack{x=0 \\
u=0}}=\left.\left[\begin{array}{ccc}
\frac{\partial f_{1}}{\partial u_{1}} & \ldots & \frac{\partial f_{1}}{\partial u_{m}} \\
\ldots \ldots & \ldots & \ldots \ldots \\
\frac{\partial f_{n}}{\partial u_{1}} & \cdots & \frac{\partial f_{n}}{\partial u_{m}}
\end{array}\right]\right|_{\substack{x=0 \\
u=0}}, \\
& C(t)=\left.\frac{\partial f}{\partial x}\right|_{\substack{x=0 \\
u=0}}=\left.\left[\begin{array}{ccc}
\frac{\partial h_{1}}{\partial x_{1}} & \ldots & \frac{\partial h_{1}}{\partial x_{n}} \\
\ldots \ldots & \ldots & \ldots \ldots \\
\frac{\partial h_{p}}{\partial x_{1}} & \ldots & \frac{\partial h_{p}}{\partial x_{n}}
\end{array}\right]\right|_{\substack{x=0 \\
u=0}}, \\
& D(t)=\left.\frac{\partial f}{\partial x}\right|_{\substack{x=0 \\
u=0}}=\left.\left[\begin{array}{ccc}
\frac{\partial h_{1}}{\partial u_{1}} & \cdots & \frac{\partial h_{1}}{\partial u_{m}} \\
\ldots \ldots & \ldots & \ldots \ldots \\
\frac{\partial h_{p}}{\partial u_{1}} & \cdots & \frac{\partial h_{p}}{\partial u_{m}}
\end{array}\right]\right|_{\substack{x=0 \\
u=0}},
\end{aligned}
$$

$N_{f}(x, u, t)$ and $N_{h}(x, u, t)$ are the nonlinear parts of $f(x, u, t)$ and $h(x, u, t)$, respectively, and

$$
\begin{array}{r}
\lim _{\|x\|,\|u\| \rightarrow 0} \frac{N_{f}(x, u, t)}{\|x\|\|u\|}=0, \\
\lim _{\|x\|,\|u\| \rightarrow 0} \frac{N_{h}(x, u, t)}{\|x\|\|u\|}=0 .
\end{array}
$$

The linear system

$$
\begin{aligned}
& \dot{x}=A(t) x+B(t) u, \\
& y=C(t) x+D(t) u
\end{aligned}
$$

is called a linear approximation of the nonlinear system (1) in the neighborhood of zero $(x=0, u=0)$.

Example 1. Consider the nonlinear system

$$
\begin{aligned}
\dot{x}_{1} & =x_{1} t+x_{2}+\sin x_{2}^{2}+u+u^{2}, \\
\dot{x}_{2} & =x_{2} e^{x_{1}}+x_{2}+2 u, \\
y & =x_{1}+u t+u^{3} .
\end{aligned}
$$

Using (5), we obtain

$$
A(t)=\left.\left[\begin{array}{ll}
\frac{\partial f_{1}}{\partial x_{1}} & \frac{\partial f_{1}}{\partial x_{2}} \\
\frac{\partial f_{2}}{\partial x_{1}} & \frac{\partial f_{2}}{\partial x_{2}}
\end{array}\right]\right|_{\substack{x=0 \\
u=0}}=\left[\begin{array}{ll}
t & 1 \\
0 & 1
\end{array}\right],
$$

$$
\begin{aligned}
& B(t)=\left.\left[\begin{array}{c}
\frac{\partial f_{1}}{\partial u} \\
\frac{\partial f_{2}}{\partial u}
\end{array}\right]\right|_{\substack{x=0 \\
u=0}}=\left[\begin{array}{l}
1 \\
2
\end{array}\right], \\
& C(t)=\left.\left[\frac{\partial h}{\partial x_{1}} \frac{\partial h}{\partial x_{2}}\right]\right|_{\substack{x=0 \\
u=0}}=[10], \\
& D(t)=\left.\left[\frac{\partial h}{\partial u}\right]\right|_{\begin{array}{l}
x=0 \\
u=0
\end{array}}=[t]
\end{aligned}
$$

and

$$
\begin{aligned}
N_{f}(x, u, t) & =\left[\begin{array}{c}
f_{1}(x, u, t) \\
f_{2}(x, u, t)
\end{array}\right]-A(t) x-B(t) u \\
& =\left[\begin{array}{c}
\sin x_{2}^{2}+u^{2} \\
x_{2} e^{x_{1}}
\end{array}\right], \\
N_{h}(x, u, t) & =h(x, u, t)-C(t) x-D(t) u=u^{3} .
\end{aligned}
$$

It is easy to check that the functions in (10) satisfy the conditions (6).

\section{Main Result}

Lemma 1. Let

$$
\dot{x}=A(t) x
$$

be the linear approximation of the nonlinear autonomous system

$$
\dot{x}=f(x, t)=A(t) x+N_{f}(x, t),
$$


where

$$
\begin{aligned}
A(t) & =\left[a_{i j}(t)\right]_{\substack{i=1, \ldots, n \\
j=1, \ldots, n}}=\left.\frac{\partial f}{\partial x}\right|_{x=0} \\
& =\left[\begin{array}{lll}
\frac{\partial f_{1}}{\partial x_{1}} & \cdots & \frac{\partial f_{1}}{\partial x_{n}} \\
\frac{\partial f_{n}}{\partial x_{1}} & \cdots & \frac{\partial f_{n}}{\partial x_{n}}
\end{array}\right]_{x=0}
\end{aligned}
$$

and

$$
\lim _{\|x\| \rightarrow 0} \frac{N_{f}(x, t)}{\|x\|}=0 .
$$

If the components $f_{1}(x, t), \ldots, f_{n}(x, t)$ of $f(x, t)$ satisfy the condition

$$
f_{i}(x, t) \geq 0
$$

for $x_{j} \geq 0, i \neq j, x_{i}=0$ and all $t \geq 0$, then

$$
a_{i j}(t) \geq 0
$$

for $i \neq j$ and all $t \geq 0$, where $i, j=1, \ldots, n$.

Proof. From (13) we have

$$
\begin{aligned}
a_{i j}(t) & =\left.\left[\frac{\partial f_{i}(x, t)}{\partial x_{j}}\right]\right|_{x=0} \\
& =\lim _{x_{j} \rightarrow 0^{+}} \frac{f_{i}\left(0, \ldots, 0, x_{j}, 0, \ldots, 0, t\right)-f_{i}(0,0, \ldots, 0, t)}{x_{j}} \\
& =\lim _{x_{j} \rightarrow 0^{+}} \frac{f_{i}\left(0, \ldots, 0, x_{j}, 0, \ldots, 0, t\right)}{x_{j}} \geq 0
\end{aligned}
$$

since $f_{i}\left(0, \ldots, 0, x_{j}, 0, \ldots, 0, t\right) \geq 0$.

Remark 1. In the particular case when $f(x, t)$ is explicitly independent of time $t, f(x, t)=f(x)$, we have $A(t)=A$ and the time-invariant matrix (13) is a Metzler matrix satisfying the condition $e^{A t} \in \mathbb{R}_{+}^{n \times n}$ for all $t \geq 0$.

Definition 1. The nonlinear system (1) is called locally positive in the neighborhood of zero $(x=0, u=0)$ if there exists a neighbourhood of the zero $U_{0}$ such that for any $x_{0} \in U_{0} \cap \mathbb{R}_{+}^{n}$ we have $x(t) \in U_{0} \cap \mathbb{R}_{+}^{n}$ for $t \geq 0$ (or at least $t \in[0, \varepsilon)$ for some $\varepsilon>0$ ).

Theorem 1. The nonlinear system (1) is locally positive in the neighborhood of zero $(x=0, u=0)$ if and only if

$$
\int_{0}^{t} \frac{\partial f_{i}}{\partial x_{j}}(\tau) \mathrm{d} \tau \geq 0
$$

for $i \neq j, i, j=1, \ldots, n$ and $t \geq 0$,

$$
\begin{aligned}
& \left.\frac{\partial f}{\partial u}(t)\right|_{\substack{x=0 \\
u=0}} \in \mathbb{R}_{+}^{n \times m},\left.\quad \frac{\partial h}{\partial x}(t)\right|_{\begin{array}{l}
x=0 \\
u=0
\end{array}} \in \mathbb{R}_{+}^{p \times n}, \\
& \left.\frac{\partial h}{\partial u}(t)\right|_{\begin{array}{l}
x=0 \\
u=0
\end{array}} \in \mathbb{R}_{+}^{p \times m}
\end{aligned}
$$

for $t \geq 0$.
Proof. Note that the condition (18a) is equivalent to

$$
\int_{0}^{t} a_{i j}(\tau) \mathrm{d} \tau \geq 0
$$

for $i \neq j, i, j=1, \ldots, n$ and $t \geq 0$. In (Kaczorek, 2001) it was shown that the linear approximation (7) is positive if and only if the conditions (19) and (18b) are satisfied. Thus, based on (6) it is easy to show that the nonlinear system (1) is locally positive in the neighborhood of zero if and only if the linear approximation (7) is positive.

Example 2. (Continuation of Example 1) We shall show that the nonlinear system (8) is locally positive in the neighborhood of zero $(x=0, u=0)$.

The nonlinear system (8) satisfies the conditions (18) since

$$
\begin{aligned}
& \int_{0}^{t} \frac{\partial f_{1}}{\partial x_{1}}(\tau) \mathrm{d} \tau=\int_{0}^{t} \tau \mathrm{d} \tau \geq 0, \\
& \int_{0}^{t} \frac{\partial f_{1}}{\partial x_{2}}(\tau) \mathrm{d} \tau=\int_{0}^{t} 1 \mathrm{~d} \tau \geq 0, \\
& \int_{0}^{t} \frac{\partial f_{2}}{\partial x_{2}}(\tau) \mathrm{d} \tau=\int_{0}^{t} 0 \mathrm{~d} \tau=0, \\
& \int_{0}^{t} \frac{\partial f_{2}}{\partial x_{2}}(\tau) \mathrm{d} \tau=\int_{0}^{t} 1 \mathrm{~d} \tau \geq 0 \text { for } t \geq 0
\end{aligned}
$$

and

$$
C(t)=\left[\begin{array}{ll}
1 & 0
\end{array}\right] \in \mathbb{R}_{+}^{1 \times 2}, \quad D(t)=t \geq 0 .
$$

Therefore, by Theorem 1 the nonlinear system (8) is locally positive in the neighborhood of zero.

Let $C_{+} \in \mathbb{R}_{+}^{n}$ be a cone in the neighborhood of zero $(x=0, u=0)$. Following Walczak (1990), the notion of local reachability in the direction of a cone will be introduced.

Definition 2. The nonlinear system (1) is called locally reachable in the cone direction of a $C_{+}$if for every state $x_{f} \in C_{+}$there exist a time $t_{f}-t_{0}>0$ and an input $u(t) \in \mathbb{R}_{+}^{m}, t \in\left[t_{0}, t_{f}\right]$ such that $x\left(t_{f}\right)=x_{f}$ for $x\left(t_{0}\right)=x_{0}=0$.

A matrix is called the monomial matrix if its every row and every column contain only one positive entry and the remaining entries are zero.

The inverse matrix $A^{-1}$ of a positive matrix $A \in$ $\mathbb{R}_{+}^{n \times n}$ is a positive matrix if and only if $A$ is a monomial matrix (Kaczorek, 2002). 
Theorem 2. The nonlinear system (1) is locally reachable in the direction of a cone $C_{+} \subset \mathbb{R}_{+}^{n}$ if the matrix

$$
R_{f}=\int_{t_{0}}^{t_{f}} \Phi\left(t_{f}, \tau\right) B(\tau) B^{T}(\tau) \Phi^{T}\left(t_{f}, \tau\right) \mathrm{d} \tau
$$

$t_{f}>t_{0}$, is a monomial matrix.

The input that steers the state of the system (1) in time $t_{f}-t_{0}$ from $x\left(t_{0}\right)=0$ to the final state $x_{f}$ is given by

$$
u(t)=B^{T}(t) \Phi\left(t_{f}, t\right) R_{f}^{-1} x_{f} \quad \text { for } t \in\left[t_{0}, t_{f}\right] .
$$

Proof. If $R_{f}$ is a monomial matrix, then there exists the inverse matrix $R_{f}^{-1} \in \mathbb{R}_{+}^{n \times n}$, which is also monomial. Hence the output (21) is well defined and $u(t) \in \mathbb{R}_{+}^{m}$ for $t \in\left[t_{0}, t_{f}\right]$.

Substituting (21) into the solution

$$
x(t)=\Phi\left(t, t_{0}\right) x_{0}+\int_{t_{0}}^{t} \Phi(t, \tau) B(\tau) \mathrm{d} \tau
$$

of (7a) for $t=t_{f}$ and $x_{0}=0$, we obtain

$$
\begin{aligned}
x\left(t_{f}\right)= & \int_{t_{0}}^{t_{f}} \Phi\left(t_{f}, \tau\right) B(\tau) B^{T}(\tau) \Phi^{T}\left(t_{f}, \tau\right) R_{f}^{-1} x_{f} \mathrm{~d} \tau \\
= & {\left[\int_{t_{0}}^{t_{f}} \Phi\left(t_{f}, \tau\right) B(\tau) B^{T}(\tau) \Phi^{T}\left(t_{f}, \tau\right) \mathrm{d} \tau\right] } \\
& \times R_{f}^{-1} x_{f}=x_{f} .
\end{aligned}
$$

If the matrix (20) is a monomial matrix, then the linear approximation (7a) of the nonlinear system (1) is reachable. Thus, based on (6) it is easy to show that the nonlinear system (1) is locally reachable in the cone $C_{+}$.

In particular, if the system (1) is linear, from Theorems 1 and 2 we obtain the known results given in (Kaczorek, 2001; 2002; Klamka, 1998; Klamka and Kalinowski, 1998).

\section{Concluding Remarks}

The notion of local positiveness in the neighborhood of zero of a nonlinear time-varying system was introduced. Necessary and sufficient conditions for the local positiveness were established using the linear approximation (7) of the nonlinear system (1). The concept of local reachability in the direction of a cone of the positive nonlinear systems (1) was introduced and sufficient conditions for local reachability in the direction of a cone using the linear approximation (7) were also derived.

With minor modifications, the deliberations can be extended to nonlinear discrete time-varying systems. Extensions to singular nonlinear systems and 2D nonlinear systems are open problems.

\section{Acknowledgment}

I wish to express my thanks to Professor Stanisław Walczak for his valuable remarks and suggestions.

\section{References}

d'Alessandro P. and de Santis E. (1994): Positivness of dynamic systems with non positive coefficients matrices. - IEEE Trans. Automat. Contr., Vol. AC-39, pp. 131-134.

Berrman A., Neumann M. and Stern R. (1989): Nonnegative Matrices in Dynamic Systems. — New York: Wiley.

Berman A. and Plemmons R.J. (1994): Nonnegative Matrices in Mathematical Sciences. — Philadelphia: SIAM Press.

Caccetta L. and Rumchev V.G. (1998): Reachable discrete-time positive systems with minimal dimension control sets. Dynam. Cont. Discr. Impuls. Syst., Vol. 4, pp. 539-552.

Caccetta L. and Rumchev V.G. (2000): A survey of reachability and controllability for positive linear systems. - Ann. Opers. Res., Vol. 98, pp. 101-122.

Fanti M.P., Maione B. and Turchsano B. (1990): Controllability of multi-input positive discrete-time systems. - Int. J. Contr., Vol. 51, No. 6, pp. 1295-1308.

Farina L. and Rinaldi S. (2000): Positive Linear Systems, Theory and Applications. - New York: Wiley.

Fornasini E. and Valcher M.E. (1997): Recent developments in $2 D$ positive systems theory. - J. Appl. Math. Comp. Sci., Vol. 7, No. 4, pp. 713-735.

Gantmacher F.R. (1959): The Theory of Matrices. - Chelsea, New York.

Kaczorek T. (2001): Externally and internally positive timevarying linear systems. - Int. J. Appl. Math. Comput. Sci., Vol. 11, No. 4, pp. 957-964.

Kaczorek T. (2002): Positive $1 D$ and 2D Systems. - London: Springer-Verlag.

Kaczorek T. (1998): Weakly positive continuous-time linear systems. - Bull. Pol. Acad. Sci. Techn. Sci., Vol. 46, No. 2, pp. 233-245.

Kaczorek T. (1998): Positive descriptor discrete-time linear systems. - Probl. Nonlin. Anal. Eng. Syst., Vol. 1, No. 7, pp. 38-54. 
Klamka J. (1998): Constrained controllability of positive $2 D$ systems. - Bull. Pol. Acad. Techn. Sci., Vol. 46, No. 1, pp. 93-102.

Klamka J. and Kalinowski J. (1998): Reachability and controllability of positive continuous-time linear systems with time variable coefficients. - Bull. Pol. Acad. Techn. Sci., Vol. 49, No. 4, pp. 633-641.

Ohta Y., Madea H. and Kodama S. (1984): Reachability, observability and realizability of continuous-time positive systems. - SIAM J. Contr. Optim., Vol. 22, No. 2, pp. 171180.

Rumchev V.G. (2000): Feedback and positive feedback holdability of discrete-time systems. - Syst. Sci., Vol. 26, No. 3, pp. 15-23.

Rumchev V.G. and Anstee R.P. (1999): Asymptotic reachable sets for discrete-time positive linear systems. - Syst. Sci., Vol. 25, No. 1, pp. 41-47.

Rumchev V.G. and James D.J.G. (1995): Spectral characterisation and pole assignment for positive linear discrete-time systems. - Int. J. Syst. Sci., Vol. 26, No. 2, pp. 295-312.
Rumchev V.G. and James D.J.G. (1990): The role of nonnegative matrices in discrete-time mathematical modelling. - Int. J. Math. Educ. Sci. Technol., Vol. 21, No. 2, pp. 169-182.

Walczak S. (1984): A note on the controllability of nonlinear systems. - Math. Syst. Theory, Vol. 17, pp. 351-356.

Valcher M.E. (1996): Controllability and reachability criteria for discrete-time positive systems. - Int. J. Contr., Vol. 65, No. 3, pp. 511-536.

Valcher M.E. (1997): On the internal stability and asymptotic behaviour of $2 D$ positive systems. - IEEE Trans. Circ. Syst., Part I, Vol. 44, pp. 602-613.

Received: 25 May 2003 Revised: 8 July 2003 